\title{
Fractal Geometry of Rocks
}

\author{
A.P. Radliński, ${ }^{1}$ E. Z. Radlińska, ${ }^{2}$ M. Agamalian, ${ }^{3}$ G. D. Wignall, ${ }^{3}$ P. Lindner, ${ }^{4}$ and O. G. Randl ${ }^{4}$ \\ ${ }^{1}$ Australian Geological Survey Organization, GPO Box 378, Canberra, Australian Capital Territory 2601, Australia \\ ${ }^{2}$ Department of Applied Mathematics, The Australian National University, GPO Box 4, Canberra, \\ Australian Capital Territory 0200, Australia \\ ${ }^{3}$ Solid State Division, Oak Ridge National Laboratory, Oak Ridge, Tennessee 37831-6393 \\ ${ }^{4}$ Institut Max von Laue-Paul Langevin, 38042 Grenoble Cedex, France
}

(Received 6 August 1998)

\begin{abstract}
The analysis of small- and ultra-small-angle neutron scattering data for sedimentary rocks shows that the pore-rock fabric interface is a surface fractal $\left(D_{s}=2.82\right)$ over 3 orders of magnitude of the length scale and 10 orders of magnitude in intensity. The fractal dimension and scatterer size obtained from scanning electron microscopy image processing are consistent with neutron scattering data. [S0031-9007(99)08945-0]
\end{abstract}

PACS numbers: 61.43.Hv, 61.12.Ex, 91.65.-n

Owing to the limited size range over which the fractal properties are usually observed, the issue of the apparent fractal geometry of various natural objects is a contentious one. In their critique of 96 recent reports on the fractality of a wide range of physical systems, Avnir et al. pointed out the contradiction between the narrow range of the appropriate scaling properties for declared fractal objects (centered around 1.3 orders of magnitude) and the public image of the status of experimental fractals [1], which for rocks has previously been based on limited experimental evidence (about 1.5 decades in length scale). A notable exception is the x-ray study of Bale and Schmidt on coals (Ref. [2], 2 decades in length scale, 7.5 decades in intensity). In this study we extend the range of length scales studied for rocks to over 3 decades (10 decades in intensity) and show that sedimentary rocks are in fact one of the most extensive fractal systems found in nature.

Sedimentary rocks are formed from a mixture of organic and inorganic debris deposited in an aqueous environment, buried and compacted at elevated temperatures over geological periods of time. Remarkably, there is no percolation threshold observed in sedimentary rocks, which indicates a microstructure more complex than one originating from just a collection of compacted grains. According to the antisintering hypothesis of Cohen, the rock/pore interface evolves by maximizing the internal surface area in response to the secular equilibrium between the rock matrix and the formation brine [3]. Various studies performed on rocks of different origin and lithology over length scales in the range $20 \AA$ to $100 \mu \mathrm{m}$ have shown that sedimentary rocks are often effective fractals [4]. Experimental tools used in these studies include molecular adsorption [5], microscopic techniques [6,7], and small-angle scattering (SAS) methods. SAS methods are particularly well suited for testing the porematrix interface: They are noninvasive, average over the entire sample volume, and include correlation information. Previous small-angle neutron and $\mathrm{x}$-ray scattering (SANS and SAXS) studies on shales [8-10] and sand- stones $[7,9,11]$ demonstrated the surface fractal geometry of the pore-matrix interface in the scale range $20 \AA$ to about $2000 \AA$.

Recent progress in neutron scattering instrumentation enables one to access the microstructure of rocks well beyond the conventional SANS $Q$ limit of $Q_{\min }=3 \times$ $10^{-3} \AA^{-1}$. The 80-m SANS instrument D11 at ILL [12] has resolution $Q_{\text {min }}=8 \times 10^{-4} \AA^{-1}$ and the Bonse-Hart geometry USANS facility at ORNL [13] can probe $Q$ range down to $2.5 \times 10^{-5} \AA^{-1}$. For periodic structures this corresponds to the maximum size limit, $2 \pi / Q_{\min }$, of about $25 \mu \mathrm{m}$. For fractal systems, a full SANS characterization of a particular size range requires access to lower $Q$ values such that $Q R<1$, and the minimum size limit is about four micrometers. The advantages of extending the $Q$ range for both microstructural and geochemical applications have been demonstrated in a recent work on artificially pyrolyzed hydrocarbon source rocks [14].

In this work we used SANS instrument D11 at ILL $(\lambda=4.5,7$, and $14 \AA)$, USANS facility at ORNL $(\lambda=$ $2.59 \AA)$ and the 30-m SANS facility at ORNL $(\lambda=$ $4.75 \AA$ ) [15]. The instruments cover the $Q$ range $2.5 \times$ $10^{-5} \leq Q \leq 0.3 \AA^{-1}$, which offers an opportunity to study for the first time the microstructure of a natural rock in the continuous range of sizes $2 \mathrm{~nm} \leq R \leq 5 \mu \mathrm{m}$. In this Letter we report SANS, USANS, and SEM results for a hydrocarbon source rock U116, originating from $342.7 \mathrm{~m}$ depth in the Urapunga 4 well (Velkerri Formation, MacArthur Basin, Northern Territory, Australia [10]). Solid rock samples cut out in-bedding-plane were used and the SANS spectra were fully isotropic [10].

SEM has been a major tool used in petrography, which not only helped to visualize the complexity of the rock matrix, but also provided information used to demonstrate the fractal character of sedimentary rocks and determine their fractal dimension for the first time [6]. Although the small-angle scattering (SAS) techniques are better suited for the latter purpose, only the combination of SAS 
and microscopy provides a means to visualize the fractal structure, achieve the necessary scale coverage of the structural data (upper bound for SAS and lower bound for SEM) and cross check the results using two independent techniques.

The specific surface area of surface fractals, $\sigma$, scales with the length scale $r$ according to $\sigma=\sigma_{x} r^{2-D_{s}}$, where $D_{s}$ is the fractal dimension. The prefactor $\sigma_{x}$ can be determined from the small-angle scattering data in the large- $Q$ limit $[16,17]$ :

$$
\sigma_{x}=\frac{\lim _{Q \rightarrow \infty}\left[Q^{6-D_{s}} \frac{d \Sigma}{d \Omega}(Q)\right]}{\pi \Delta \rho^{2} \rho_{0} F\left(D_{s}\right)},
$$

where $Q$ is the scattering vector, $\frac{d \Sigma}{d \Omega}(Q)$ is the scattering cross section, $\Delta \rho$ is the scattering length density contrast, $\rho_{0}$ is mass density, and $F\left(D_{s}\right)=\frac{\Gamma\left(5-D_{s}\right) \sin \left[\left(3-D_{s}\right)(\pi / 2)\right]}{\left(3-D_{s}\right)}$. In the large- $Q$ limit, $\frac{d \Sigma}{d \Omega}(Q)$ measured in the scattering experiment has the asymptotic form $[8,18]$ :

$$
\frac{d \Sigma}{d \Omega}(Q) \rightarrow A\left(D_{s}\right) \times Q^{D_{s}-6}+\ldots
$$

from which the surface fractal dimension, $2<D_{s} \leq 3$, can be directly determined. The amplitude, as calculated by Bale and Schmidt [2] and modified by Wong and Bray [17], is $A(D)=\pi I_{0} \Delta \rho^{2} \sigma_{x} \rho_{0} V F\left(D_{s}\right)$, where $I_{0}$ is a constant determined by the incident intensity and $V$ is the sample volume. Real fractal objects scatter according to Eq. (2) only within a limited $Q$ range. Assuming that the density-density correlation function decays exponentially above the upper size limit $\xi$ of the fractal object, the following result is obtained [8]:

$$
\begin{aligned}
\frac{d \Sigma}{d \Omega}(Q) \propto & Q^{-1} \Gamma\left(5-D_{s}\right) \xi^{5-D_{s}}\left[1+(Q \xi)^{2}\right]^{\left(D_{s}-5\right) / 2} \\
& \times \sin \left[\left(D_{s}-1\right) \arctan (Q \xi)\right],
\end{aligned}
$$

which reduces to Eq. (2) in the large- $Q$ limit, but saturates in the region $Q \xi \gg 1$. Since shales, even with significant organic matter content, are perceived by neutrons as two phase [10], the two-phase approximation inherent in Eqs. (2) and (3) can be applied to interpret SANS data for organic-rich sedimentary rocks.

Behavior described by Eq. (3) is qualitatively similar to the distortion of fractal scattering by the multiple scattering (MS) effects [19]. In order to test for MS and refraction effects [20] (not detected), our preliminary measurements were performed on samples of several thicknesses. The SANS curves obtained with instrument D11 using long-wavelength neutrons $(\lambda=14 \AA)$ show significant dependence on sample thickness (Fig. 1), which indicates pronounced MS effects. For strongly scattering sedimentary rocks MS may be particularly misleading in the small- $Q$ region, where there is a similarity between the saturation caused by MS and the flattening out due to the finite size of fractal scatterers [Eq. (3)].

In this study it was possible to thin down rock samples until MS became irrelevant. Figure 2 shows

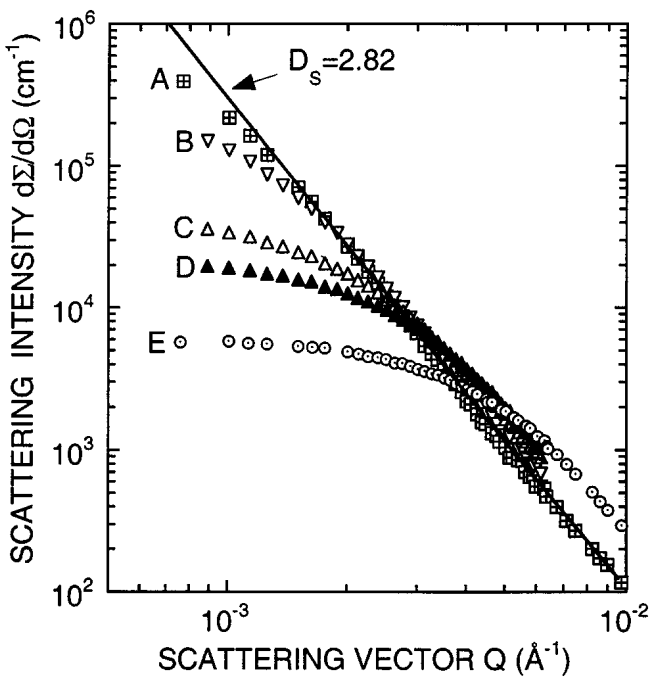

FIG. 1. SANS data acquired for various sample thicknesses (instrument D11, $\lambda=14 \AA$ A). (A) $t=0.63 \mathrm{~mm}$; (B) $t=$ $1.20 \mathrm{~mm}$; (C) $t=3.09 \mathrm{~mm}$; (D) $t=4.23 \mathrm{~mm}$; and (E) $t=$ $7.4 \mathrm{~mm}$.

the absolute scattering cross section for a $0.1 \mathrm{~mm}$ thick sample measured at three different neutron wavelengths: $4.5,7$, and $14 \AA$. The three experimental curves coincide in the overlapping $Q$ range. MS is evidently absent as its contribution, inversely proportional to $\lambda^{2}$, would otherwise deform different experimental curves to a different degree. The SANS experiments at $\lambda \simeq 5 \AA$ and USANS experiments at $\lambda=2.59 \AA$ were performed using samples about $1 \mathrm{~mm}$ thick with no significant contribution from MS.

Figure 3 shows the absolute scattering cross section (in $\mathrm{cm}^{-1}$ ) for rock U116, calculated from the combined SANS and USANS data. The experimental curve shown in Fig. 3 represents the single-scattering cross section. The ORNL and ILL SANS data were calibrated

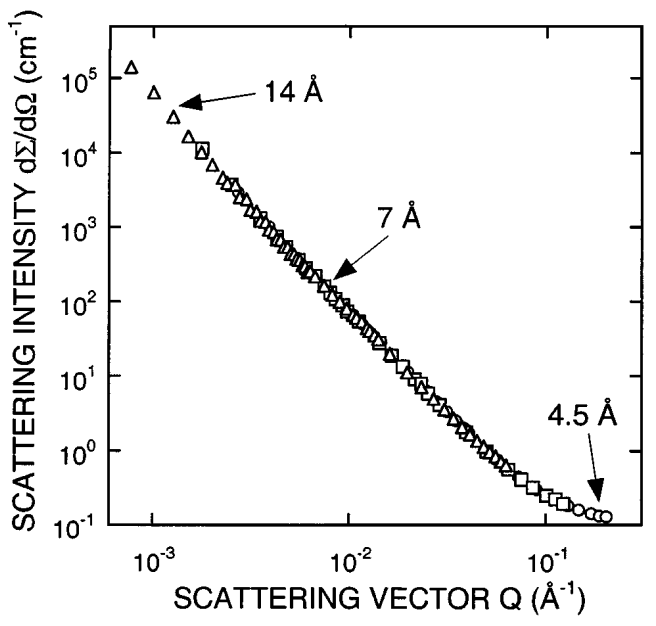

FIG. 2. SANS data acquired from a thin sample $(t=0.1 \mathrm{~mm})$ using various neutron wavelengths. Instrument D11: $\lambda=4.5 \AA$ (circles), $\lambda=7 \AA$ (squares), and $\lambda=14 \AA$ (triangles). 


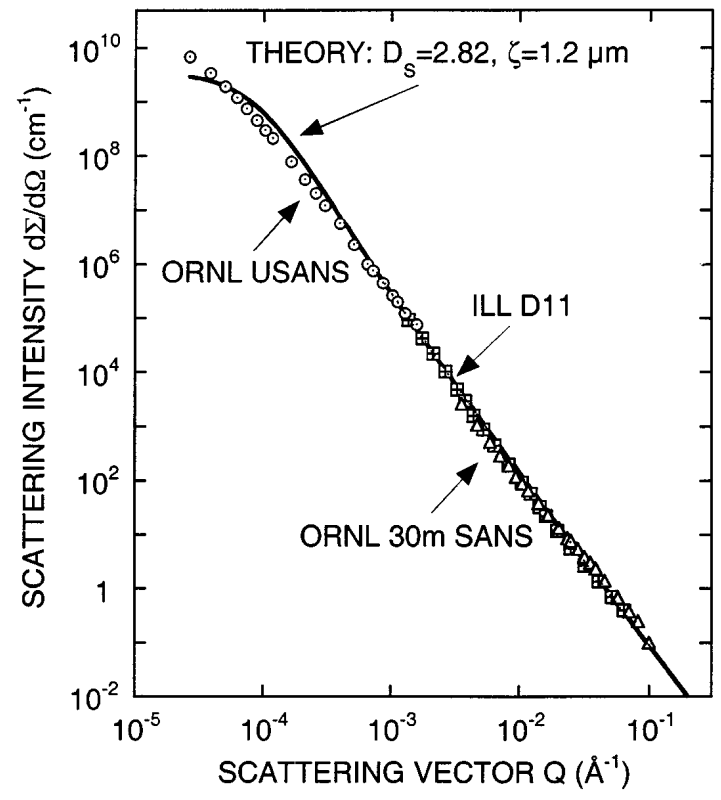

FIG. 3. Absolute neutron scattering cross section for rock U116. Experimental errors are less than the symbol size.

independently with no adjustable scale factors $[21,22]$ and an excellent agreement was obtained. A flat scattering background of $0.13 \mathrm{~cm}^{-1}$ (Fig. 2) was subtracted from SANS experimental values. This value is most probably dominated by the small-scale inhomogeneities, since the estimated incoherent scattering cross section on hydrogen nuclei present in the organic matter and in formation waters is only about $0.02 \mathrm{~cm}^{-1}$. USANS data have been transformed to the point geometry by the means of Lake technique [23] using formula (3) for fitting the experimental curves measured in the slit geometry.

The region of the power-law scattering in Fig. 3 extends over 3 orders of magnitude of the length scale $(6 \mathrm{~nm} \leq 2 \pi / Q \leq 6 \mu \mathrm{m})$ and 10 orders of magnitude of the scattering cross section $\left(10^{-1} \leq d \Sigma / d \Omega \leq\right.$ $\left.10^{9} \mathrm{~cm}^{-1}\right)$. Such an extent of fractal microstructure in a rock is remarkable, in particular when compared with numerous other reports on the fractal properties of natural systems [1]. The slope of -3.18 obtained from a straightline fit in the $10^{-4} \leq Q \leq 10^{-1} \AA^{-1}$ region corresponds to a surface fractal of dimension $D_{s}=2.82$, which is within the range of fractal dimensions found previously for Urapunga 4 source rocks [10]. From experimental data we obtain $\lim _{Q \rightarrow \infty}\left[Q^{6-D_{s} \frac{d \Sigma}{d \Omega}}(Q)\right]=6 \times 10^{-5} \AA^{D-6} \mathrm{~cm}^{-1}$. Substituting to Eq. (1) $\Delta \rho^{2}=8.41 \times 10^{20} \mathrm{~cm}^{-4}$ and $\rho_{0}=2.45 \mathrm{~g} / \mathrm{cm}^{3}$ [10], we obtain the specific surface area for coverage with nitrogen gas (molecular crosssectional area $16.2 \AA^{2}$, Ref. [16]) $\sigma(4 \AA)=17.5 \mathrm{~m}^{2} / \mathrm{g}$. This is within the range of the specific surface areas for shales determined using molecular adsorption techniques, which vary from 10 to $60 \mathrm{~m}^{2} / \mathrm{g}$ between depths 500-3500 m [24].

The departure of the scattering curve from straight line in the ultra-small- $Q$ region is real, although minimal.
The shape of scattering curve in this region varies for samples originating from various depths in the Urapunga 4 core, indicating differences in the large-scale structure of scatterers at various depths of burial. The solid curve calculated for a single fractal component [formula (3), $\xi=1.2 \mu \mathrm{m}$ and $\left.D_{s}=2.82\right]$ is shown for illustration in Fig. 3. It was not possible to obtain a good fit to formula (3) over the entire $Q$ range, and it is likely that there is more than one fractal component in the system. The value of $\xi$ for rock U116 could only be estimated to be of the order of several $\mu \mathrm{m}$.

SEM images of the surface of sample U116 cleaved inbedding-plane are shown in Fig. 4. The surface texture is dominated by the illite clay particles and appears rough at any length scale. For smallest magnification, however, one can see images of roughly spherical objects about $10-20 \mu \mathrm{m}$ in diameter (Fig. 4A). Image $\mathrm{C}$ has been obtained from image A by digital enhancement (increasing contrast) of the outlines of some of these objects and serves as a guide for the eye only. The object size is close to the value of $\xi$ estimated from small-angle scattering and, therefore, it is possible that these objects are images of individual fractal scatterers.

In order to independently estimate the fractal dimension and cutoff length $\xi$ in sample U116 we used the manual "feature" counting technique [6]. For surface fractals one expects a power-law relationship between the average number of "features" per unit length, $N / L$, and the "feature size," $R: N(R) / L=$ const $\times R^{2-D_{s 1}}$, where $D_{s 1}$ is the fractal dimension characterizing the analyzed one-dimensional region. It has been argued that for high porosity rocks, which break up mostly along the preexisting pore-matrix interface, the fractal dimension measured by SANS $\left(D_{s}\right)$ and that obtained from the image analysis of cleaved rock surface $\left(D_{s 1}\right)$ probe the same structural features [6] and, therefore, should be identical. The feature counting results illustrated in Fig. 5

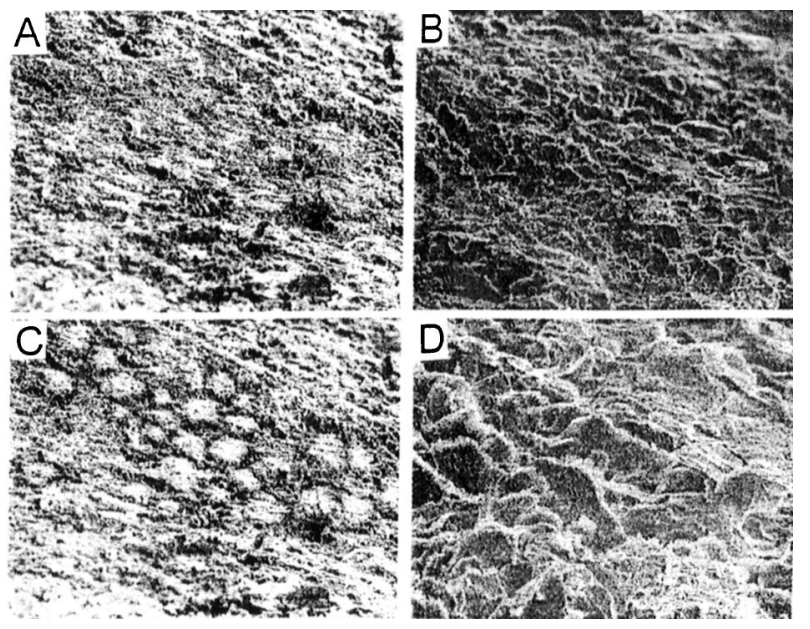

FIG. 4. SEM images for U116 samples cleaved in-bedding plane. (A) Magnification $\times 1000$; (B) magnification $\times 3000$; (C) digitally enhanced image (A); (D) magnification $\times 10000$. 


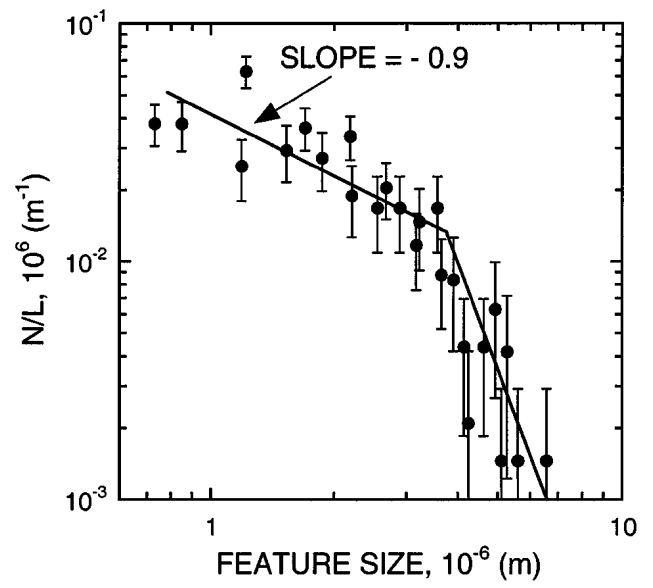

FIG. 5. Variation of the average number of "features" with feature size obtained from SEM images.

are consistent with the conclusions based on neutron scattering data. $D_{s 1}$ is about 2.8 to 2.9 for length scales smaller than about $4 \mu \mathrm{m}$ and, importantly, there is a marked dropoff of the number of features for length scales above $4 \mu \mathrm{m}$, indicating a breakdown of the fractal properties which gives an estimated value of $\xi \approx 4 \mu \mathrm{m}$. This is consistent with several $\mu \mathrm{m}$ estimated from the onset of cutoff effects in USANS data.

In conclusion, using SANS and USANS we demonstrated that the pore-matrix interface in a natural hydrocarbon source rock is a surface fractal over three decades of the length scale, from $6 \mathrm{~nm}$ to about $4 \mu \mathrm{m}$. Such an extent of the fractal properties in a natural system is remarkable. In the region 0.7 to $7 \mu \mathrm{m}$ the fractal analysis based on neutron scattering data is consistent with SEM image processing results for the same rock sample.

We acknowledge the neutron beam time grant on instrument D11 at Institut Laue-Langevin, Grenoble, France. The research at Oak Ridge was supported by the Division of Materials Science, U.S. Department of Energy under Contract No. DE-AC05-96OR22464 with Lockheed Martin Energy Research Corporation. This research was supported in part jointly by the Oak Ridge National Laboratory and the Oak Ridge Institute for Science and Education. A.P. Radliński publishes with the permission of the Executive Director, AGSO.
[1] D. Avnir, O. Biham, D. Lidar, and O. Malcai, Science 279, 39 (1997).

[2] H. D. Bale and P. W. Schmidt, Phys. Rev. Lett. 53, 596 (1984).

[3] M.H. Cohen, in Physics and Chemistry of Porous Media-II, edited by Jayanth R. Banavar, Joel Koplik, and Kenneth W. Winkler, AIP Conf. Proc. No. 154 (AIP, New York, 1987).

[4] A. H. Thompson, Annu. Rev. Earth Planet. Sci. 19, 237 (1991).

[5] D. Avnir and D. Farin, J. Chem. Phys. 79, 3566 (1983).

[6] A. J. Katz and A. H. Thompson, Phys. Rev. Lett. 54, 1325 (1985).

[7] A. H. Thompson, A. J. Katz, and C. E. Krohn, Adv. Phys. 36, 625 (1987).

[8] D.F.R. Mildner and P.L. Hall, J. Phys. D 19, 1535 (1986).

[9] P.-Z. Wong, J. Howard, and J.-S. Lin, Phys. Rev. Lett. 57, 637 (1986).

[10] A.P. Radliński, C. J. Boreham, G. D. Wignall, and J.-S. Lin, Phys. Rev. B 53, 14152 (1996).

[11] P. W. Schmidt, in The Fractal Approach to Heterogeneous Chemistry, edited by D. Avnir (Wiley and Sons, New York, 1989), p. 67.

[12] P. Lindner, R.P. May, and P.A. Timmins, Physica (Amsterdam) 180B \& 181B, 967 (1992).

[13] M. Agamalian, G. D. Wignall, and R. Triolo, J. Appl. Crystallogr. 30, 345 (1997); M. Agamalian, D. K. Christen, A. R. Drews, C. J. Glinka, H. Matsuoka, and G. D. Wignall, J. Appl. Cryst. 31, 235 (1998).

[14] A. P. Radliński, C. J. Boreham, P. Lindner, O. W. Randl, G. D. Wignall, and J. Hope (to be published).

[15] W. C. Koehler, Physica (Utrecht) 137B, 320 (1986).

[16] A. J. Hurd, D. W. Schaefer, D. M. Smith, S. B. Ross, A. Le Méhauté, and S. Spooner, Phys. Rev. B 39, 9742 (1989).

[17] P.-z. Wong and A. J. Bray, J. Appl. Cryst. 21, 786 (1988).

[18] J. E. Martin, J. Appl. Cryst. 19, 25 (1986).

[19] A. J. Allen and N. F. Berk, J. Appl. Cryst. 25, 221 (1994).

[20] R. J. Weiss, Phys. Rev. 83, 379 (1951).

[21] G. D. Wignall and F. S. Bates, J. Appl. Cryst. 20, 28 (1986).

[22] ILL Internal Report No. ILL98/LI/10T, 1998.

[23] J. A. Lake, Acta Crystallogr. 23, 191 (1967).

[24] B.P. Tissot and D. H. Welte, Petroleum Formation and Occurence (Springer-Verlag, Berlin, 1984), 2nd ed., p. 307. 